Acta Crystallographica Section E

Structure Reports

Online

ISSN 1600-5368

\section{Song-Lin Li}

Department of Chemistry, Tianjin University, Tianjin 300072, People's Republic of China

Correspondence e-mail: sllitju@yahoo.com.cn

\section{1-Carboxymethylpyridinium-2-carboxylate.} Corrigendum
The authors listed in the original report by Song, Li \& Song [Acta Cryst. (2006), E62, o4656-04657] are incorrect. The correct author of the paper is given here and is the sole author of the paper. 